Revue des patrimoines

$42 \mid 2020$

Imagerie numérique et patrimoine culturel :

représentation et transmission des connaissances

\title{
Une écriture opérative du fait technique : savoirs et savoir-faire au sein du patrimoine culturel immatériel
}

An operative writing of technique: knowledge and know-how in intangible

cultural heritage

Isabelle Chave

\section{(2) OpenEdition}

Journals

Édition électronique

URL : http://journals.openedition.org/insitu/28007

DOl : 10.4000/insitu.28007

ISSN : 1630-7305

Éditeur

Ministère de la Culture

Référence électronique

Isabelle Chave, «Une écriture opérative du fait technique : savoirs et savoir-faire au sein du patrimoine culturel immatériel », In Situ [En ligne], 42 | 2020, mis en ligne le 12 juin 2020, consulté le 12 janvier 2021. URL : http://journals.openedition.org/insitu/28007 ; DOI : https://doi.org/10.4000/insitu.28007

Ce document a été généré automatiquement le 12 janvier 2021.

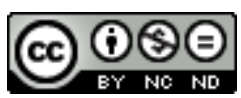

In Situ Revues des patrimoines est mis à disposition selon les termes de la licence Creative Commons Attribution - Pas d'Utilisation Commerciale - Pas de Modification 4.0 International. 


\title{
Une écriture opérative du fait technique : savoirs et savoir-faire au sein du patrimoine culturel immatériel
}

\author{
An operative writing of technique: knowledge and know-how in intangible \\ cultural heritage
}

Isabelle Chave

1 Depuis 2003 maintenant, la Convention de l'Unesco pour la sauvegarde du patrimoine culturel immatériel invite les 178 États parties, qui, comme la France en 2006, l'ont aujourd'hui ratifiée, à identifier et à décrire les connaissances, pratiques et savoir-faire à la fois traditionnels et vivants présents sur leur territoire, dans des domaines aussi variés que l'oralité, les rituels, les fêtes, les spectacles ou l'artisanat. Au gré de leur inventaire et de l'étude de leurs modes de transmission et de sauvegarde, ce paradigme patrimonial d'un nouveau genre encourage l'initiative et la participation des communautés et vise à démontrer l'apport essentiel des pratiques culturelles immatérielles au développement durable, à la cohésion sociale et au maintien de la diversité culturelle.

\section{Place et nature des savoir-faire techniques inclus à l'Inventaire national du patrimoine culturel immatériel}

2 La réalisation et la diffusion d'un inventaire des pratiques culturelles immatérielles, ou de plusieurs outils de ce type à l'échelle d'un territoire national, sont une obligation des États parties à la Convention de 2003 (art.12 et art.14). Accessible en ligne ${ }^{1}$, l'inventaire français compte 474 fiches descriptives au $1^{\mathrm{er}}$ mai 2020 , réparties en sept catégories, dont celle des savoir-faire. Depuis octobre 2017, afin de favoriser une diversification des modes de consultation et de diffusion des savoirs et leur 
déploiement dans de nouveaux espaces, ces fiches sont reversées progressivement sur une plateforme collaborative, PCI Lab, créée sur une structure de base de données et hébergée par Huma-Num, afin d'améliorer l'exposition des ressources et permettre la contribution du public, via les technologies wiki. Chaque fiche issue de l'inventaire ministériel fait l'objet d'une duplication sous forme d'une notice Wikipédia; la plateforme peut ainsi prendre en compte la capacité d'expertise, réflexive et participative, des usagers de l'Inventaire national, qui contribuent grâce au profil qu'ils se créent pour l'encyclopédie en ligne ${ }^{2}$.

Dès l'instauration de l'inventaire en 2008, au titre de la cinquième catégorie de la définition unesquienne - les «savoir-faire de l'artisanat traditionnel »-, ses fiches se sont très vite ouvertes à l'expression de savoirs techniques : savoir-faire, gestes, tours de main, art... En onze ans, 110 fiches relatives à des pratiques artisanales ont été ainsi incluses, faisant du champ spécifique des savoir-faire, avec près d'une fiche sur cinq (23\%), l'une des deux catégories les plus riches de l'Inventaire national, avec celle des jeux traditionnels. Représentatives des sujets classiques de la technologie culturelle (élevage, pêche, tissage, bâti, facture instrumentale...), ces fiches d'inventaire décrivent à la fois :

- des techniques liées à des matériaux spécifiques, tels que le bois (fabrication de carcasses de sièges à Liffol-le-Grand ou de chaises à Came, savoir-faire du tourneur sur bois, fabrication des makhilas, fabrication des quilles dans les Pyrénées, fabrication des palas, art du formaire, ou fabrication des formes de bois pour les papetiers traditionnels), le verre (cristal à Daum, art de la perle de verre, gestes des métiers d'art verriers), le métal (damassage en Lorraine, gravure héraldique, héliogravure, dorure sur métal en Bretagne, pendulerie, gravure de poinçons typographiques, mécanique horlogère, émail sur métal), le cuir (ganterie de peau et mégisserie à Saint-Junien, sellerie-maroquinerie dans le Bordelais, botterie d'équitation à Saumur, reliure-dorure à Périgueux), le végétal (rempaillage et cannage de chaises à Forcalquier, récolte du goémon ou ramassage des simples en Bretagne, savoir-faire liés aux parfums en pays de Grasse, vannerie en Bretagne - paniers de Mayun, sklisseneù, baskodenn, mann du Trégor, bosselles de Redon, carbassons, vannerie à montants en méridien -, récolte des plantes médicinales et culture du chanvre textile en Briançonnais, savoirs naturalistes de Larrau, construction de haies vives au Pays basque, vannerie du vacoa à La Réunion, fabrication des chisteras) ou la corne naturelle (fabrication de peignes) ;

- des techniques agricoles et agropastorales: élevage du bœuf gras bazadais, parcours journalier dans les Pyrénées, feu pastoral des estives basques, transformation des canards gras en Aquitaine, élevage et production de laine dans le Briançonnais, tue-cochon, savoirfaire liés à l'irrigation gravitaire par béals en Lozère ;

- des techniques alimentaires et culinaires: cultures du raisin chasselas de Moissac, du cresson à Méréville ou de l'ail de Billom; fabrication du fromage de Salers, fabrication des sugelli, salaisons fumées au tuyé du Haut-Doubs, parmi d'autres ;

- des techniques du bâti : remplissage des maisons en pan de bois, fabrication d'épis de faîtage et menuiserie-charpente du bâti ancien en Normandie, tracé de charpente, lauzerie à SaintGeniès, art de la construction en pierre sèche, fabrication de la chaux dans le Briançonnais, savoir-faire du couvreur zingueur parisien, savoir-faire de la fondation Notre-Dame de la cathédrale de Strasbourg, savoir-faire constructifs de la terre cuite ;

- des techniques maritimes et halieutiques : barquette marseillaise, pêcheries fixes du littoral de la Manche, yole ronde de la Martinique, pêche aux poissons d'eau douce des étangs de la Dombes, savoir-faire des pêcheurs du lac de Grand-Lieu ; 
- des techniques artistiques : enluminure, calligraphie, glyptique, peinture sur porcelaine à Saint-Junien, miniature au "blanc Limoges", émaillerie et porcelaine de Limoges, fabrication des émaux de Longwy, céramique d'art au Château et à Saint-André-le-Désert, faïencerie d'art de Malicorne, dorure sur bois à Chapaize, restauration d'œuvres peintes, art tembe sculpté ou peint ;

- des techniques textiles et vestimentaires : dentelle au fuseau du Puy-en-Velay, dentelle au point d'Alençon, tapisserie d'Aubusson, tissage à bras, art du tailleur à Paris, filature à Pelletin, fabrication de pigments à Bécherel, savoir-faire du fourreur, corsetterie, confection des costumes traditionnels ossalois, boutis ou broderie de Marseille, savoir-faire des éventaillistes, broderie au point de Lunéville ;

- des techniques de la facture instrumentale: facture d'instruments anciens à clavier, fabrication de sonnailles à Bourdettes, facture-restauration instrumentale à Donzy-lePertuis, fabrication du tambourin à cordes et de la cornemuse de Gascogne, fabrication du xare, facture de la flûte à trois trous, fabrication de la bombarde ou biniou.

4 À l'exception des dernières fiches incluses depuis 2017 (savoir-faire des cathédrales, savoir-faire du couvreur-zingueur parisien, gravure de poinçons typographiques, artisanat de la perle de verre, art du formaire...), qui correspondent davantage à une saisine spontanée du département ministériel du Pilotage de la recherche par des communautés de praticiens, la constitution de ce corpus s'est faite par vagues, en fonction de programmes de recherche pluriannuels résultant d'appels à projets successivement lancés : Francesca Cominelli (2008) et Lamia Gabriel (2009-2010) pour un programme "Métiers d'art » conçu en partenariat avec la Société d'encouragement aux métiers d'art (Sema), actuelle Institut national des métiers d'art (INMA) ; François Calame (2009), sur les savoirs de la charpente; l'université de Bretagne occidentale (2012), sur les métiers et savoir-faire liés à la nature du territoire d'Oust et Vilaine ; Roger Hérisset (2013), sur les savoir-faire des vanniers en Bretagne ; le PNR du Golfe du Morbihan et le Syndicat intercommunal d'aménagement du Golfe du Morbihan (2013-2014) sur les métiers et savoir-faire traditionnels du golfe du Morbihan; l'association Epinoia (2014), sur les pratiques techniques liées au développement durable dans le Briançonnais; la Fédération des sites remarquables du goût (depuis 2015), sur les techniques de culture et d'élevage; l'association Copage (2017), sur l'irrigation gravitaire en Lozère ; l'université de Paris 1 (2018) pour les savoir-faire de la maréchalerie; le Centre français du PCI, aux côtés du Syndicat professionnel des émailleurs français (2019), pour les savoir-faire de l'émail sur métal, parmi tant d'autres...

\section{Caractériser la description des techniques dans le champ du PCI}

5 Au-delà des ressources pour les praticiens et chercheurs qui s'intéressent aux savoirs et aux savoir-faire sur le terrain, l'inclusion d'un élément dans un inventaire est conçue par l'Unesco comme l'une des dimensions primordiales de la politique de sauvegarde du PCI par un État partie. Placer ainsi les pratiques culturelles immatérielles en régime d'objet constitue la clé de voûte de la politique de mise en œuvre de la Convention de 2003, si l'on fait de la transmission la notion centrale du processus de sauvegarde : "L'élaboration et la mise à jour des inventaires sont un processus continu visant à assurer l'identification en vue de garantir la transmission du PCI» (art.11 de la 
Convention). Sur proposition spontanée des communautés de praticiens ou dans le cadre d'une réponse à l'appel à projet annuel en recherche appliquée, proposé par le ministère de la Culture depuis 2013, le processus, pour les descripteurs (praticiens euxmêmes, unités de recherche, centres de ressources ou services patrimoniaux), va de la sélection d'un thème à l'observation des techniques et à la diffusion d'une fiche de synthèse des techniques observées in situ.

Les études conduites pour la réalisation de ces fiches de l'Inventaire national ont en commun plusieurs aspects :

- une sélection des pratiques techniques éligibles au regard des stipulations de la Convention de 2003, qui distinguent plusieurs critères dirimants (commercialisation excessive, impacts sur l'environnement et sur le développement durable, violence vis-à-vis de l'animal, absence de créativité humaine...) ;

- une démarche d'inventaire appuyée sur les méthodes de l'anthropologie et de l'ethnographie, qui décrit les savoirs, les techniques et les processus par le prisme des communautés impliquées ;

- l'association systématique des professionnels, des entreprises et/ou des associations spécialisées dans le secteur concerné, acceptant d'offrir un terrain de recherche ;

- l'importance donnée à la viabilité des pratiques, aux conditions de transmission, à l'éducation formelle et non formelle, aux parcours de formation et aux dispositifs éventuels de certification professionnelle, induisant la collaboration d'organismes spécialisés à l'élaboration de ces inventaires.

Figure 1
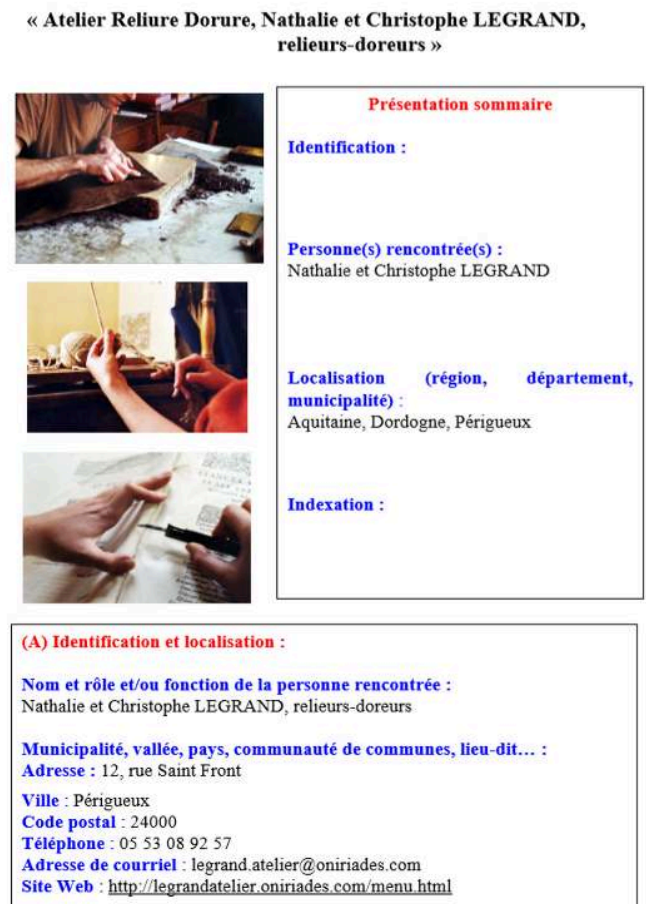

Première page illustrée de la fiche " La reliure-dorure (atelier Nathalie et Christophe Legrand) », Inventaire national du patrimoine culturel immatériel, Paris, ministère de la Culture, 2010.

Photo : Christophe Legrand. 
Instauré en 2007-2008 par la Mission de l'ethnologie du ministère de la Culture, le modèle de fiche d'inventaire utilisée pour l'inventaire français s'est inspiré des expériences menées, à l'Université Laval à Québec, par Jean Du Berger et Laurier Turgeon. Sa structure a été actualisée en 2012, en mars 2017, puis à nouveau en 2019, en particulier pour donner une plus grande place aux mesures de sauvegarde.

8 Dans chaque fiche, introduite, depuis la création de l'Inventaire national, par trois vues, toujours sélectionnées par les praticiens [fig.1], dont le rôle est de synthétiser les gestes et savoir-faire emblématiques de la pratique décrite, la description proprement dite de la technique étudiée se répartit en deux champs (cf. Annexe 1) : la partie «I. 5. Description détaillée ", limitée à 30000 caractères, et la partie «I.7. Éléments matériels liés à la pratique", qui permettent d'évoquer aussi, conformément à l'article 2 de la Convention de 2003, les vecteurs et supports des pratiques immatérielles (éléments du bâti ${ }^{3}$ ou objets, outils et matériaux). La présentation de l'aménagement de l'atelier et de ses équipements spécialisés, quand elle n'est pas omise, est le plus souvent synthétisée par une vue photographique d'ensemble, comme dans les fiches « La gravure des poinçons typographiques » [fig. 2] et «L'artisanat de la perle de verre en France » [fig. 3].

Figure 2

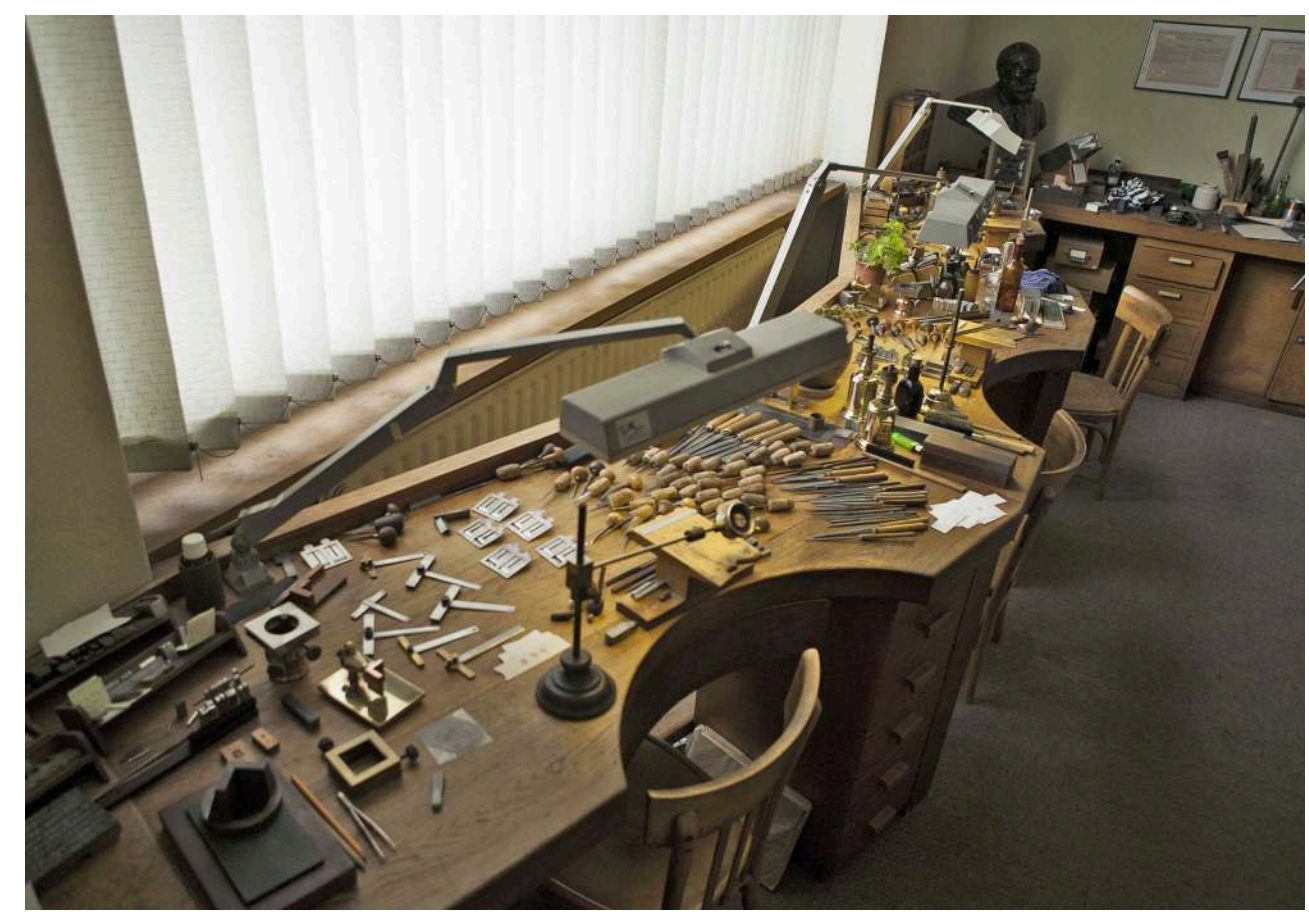

Établi de graveur de Nelly Gable. Vue extraite de la fiche "La gravure des poinçons typographiques », Inventaire national du patrimoine culturel immatériel, Paris, ministère de la Culture, 2018, p. 1

Photo : Daniel Pype / cliché : Imprimerie nationale. 
Figure 3

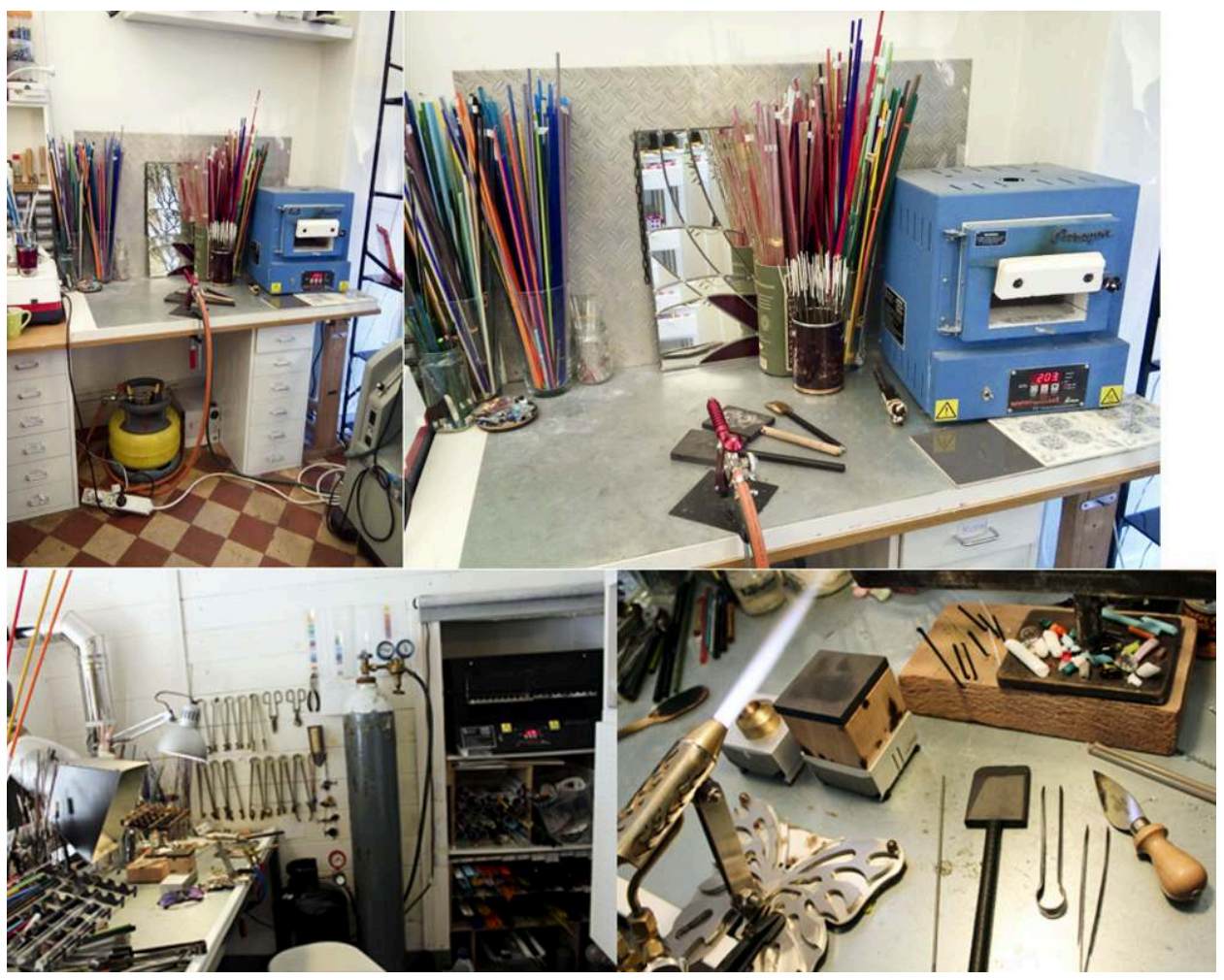

En haut et en bas: deux ateliers-type de perliers et leurs outils-type (chalumeau, moule de $18 \mathrm{~mm}$, plaque graphite, pinces et couteau à lame fine). Vues extraite de la fiche "L'artisanat de la perle de verre en France », Inventaire national du patrimoine culturel immatériel, Paris, ministère de la Culture, 2018, p. 5

Photos (en haut) : N. Srour; photos (en bas) : A. Bayens.

La part la plus importante du champ «Description détaillée» est prise par l'énumération descriptive de chacune des étapes de la châne opératoire. 
LES SAVOIR-FAIRE DU COUVREUR ZINGUEUR PARISIEN

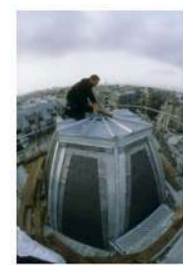

Crédilis : Gilles Mermet.

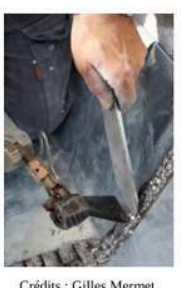

Crédits : Gilles Mermet.

Présentation sommaire

Sur les toits de Paris, le couvreur mâtrise la pose de tous les matériaux traditionnels associés à l'architecture parisienne : le zinc, le plomb, le cuivre, l'ardoise et la tuile plate, Certains de
ces matériaux sont également unilisés dans, d'autres villes de France, et ce, depuis plusieurs siécles. Mais c'est à Paris, dans la seconde moitié du XIX' siècle, que s'est généralisée l'uutilisation du zinc, pour couvrir les toits dits à la Manson des dirans de nilliers de nouveaux immeubles haussmanniens

Tapóón III en entre la découverte du laminage du zinc, d'une part, et la volonté de Napoleon III et d' 'Haussmann de reconstruire Paris, d'autre part, a donné naissance au métier
très parisien de couvrew zines entretenus par une communauté bien vivante.

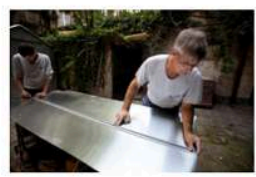

Crédits: Gilles Mermet.

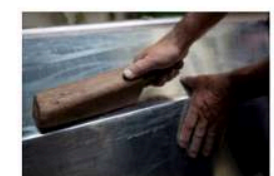

Créditis: Gilles Mermet.

Fiche « Les savoir-faire du couvreur zingueur parisien », Inventaire national du patrimoine culturel immatériel, Paris, ministère de la Culture, 2017, p. 1

9 Ainsi, dans la fiche « Les savoir-faire du couvreur zingueur parisien » [fig. 4], l'art de la pose du zinc fait-il l'objet, en sept pages, d'une description structurée en quatre techniques spécifiques, détaillée, pour l'une d'elles (la couverture à tasseaux sur terrasson), dans l'ordre logique des étapes de la réalisation ${ }^{4}$ :

«I.5.1. L'art de la pose du zinc à Paris.

- La couverture à tasseaux sur un toit à l'impériale ;

- La couverture à tasseaux sur un terrasson haussmannien :

- Le décrapouillage,

- La pose de l'isolant,

- Le voligeage,

- Le lignage et la pose des tasseaux de bois,

- Le calepinage,

- Le façonnage sur l'établi,

- La prise en compte de la dilatation,

- La pose des bacs entre les tasseaux,

- La pose des couvre-joints,

- La soudure,

- La ventilation du toit,

- Les savoir-faire à la jonction entre le zinc et d'autres matériaux ;

- La pose d'une gouttière à l'anglaise en zinc ;

- La pose des ornements en zinc ».

Pour pallier ces longs descriptifs, le recours à un tableau fonctionnel [fig. 5] a été très souvent utilisé lors de la mise au net des informations transmises par le praticien interviewé. Ce mode d'écriture en tableau a été notamment utilisé pour les fiches d'inventaire synthétiques, issues du programme de recherche sur les métiers d'art, 
conduit par Francesca Cominelli en 2008 avec la Sema, qui impliquait plus de trente métiers à inventorier en quelques mois et, au minimum, autant de praticiens à interroger sous forme d'entretiens. Par type de production, le tableau conçu organise en colonnes quatre informations: étapes de la production, description, mode d'apprentissage, nombre de personnes dédiées à cette phase.

Figure 5


Étapes du procédé d'héliogravure, établies par Fanny Boucher, chef de l'atelier Hélio'g à Meudon, spécialisé en héliogravure artisanale, et par Francesca Cominelli. Extraits de la fiche "L'héliogravure », Inventaire national du patrimoine culturel immatériel, Paris, ministère de la Culture, 2008, p. 2-3.

$\mathrm{Au}$ sein des quelque 110 fiches de pratiques techniques que compte aujourd'hui l'Inventaire national, l'absence systématique, en revanche, du recours à des schémas ou à des graphiques est particulièrement frappante. Dans les fiches les plus récentes, incluses depuis 2017, le parti pris est de recourir abondamment à la photographie, pour illustrer chaque étape des processus techniques, sans faire l'économie d'une description littérale [fig. 6 ; fig. 7]. 
Figure 6

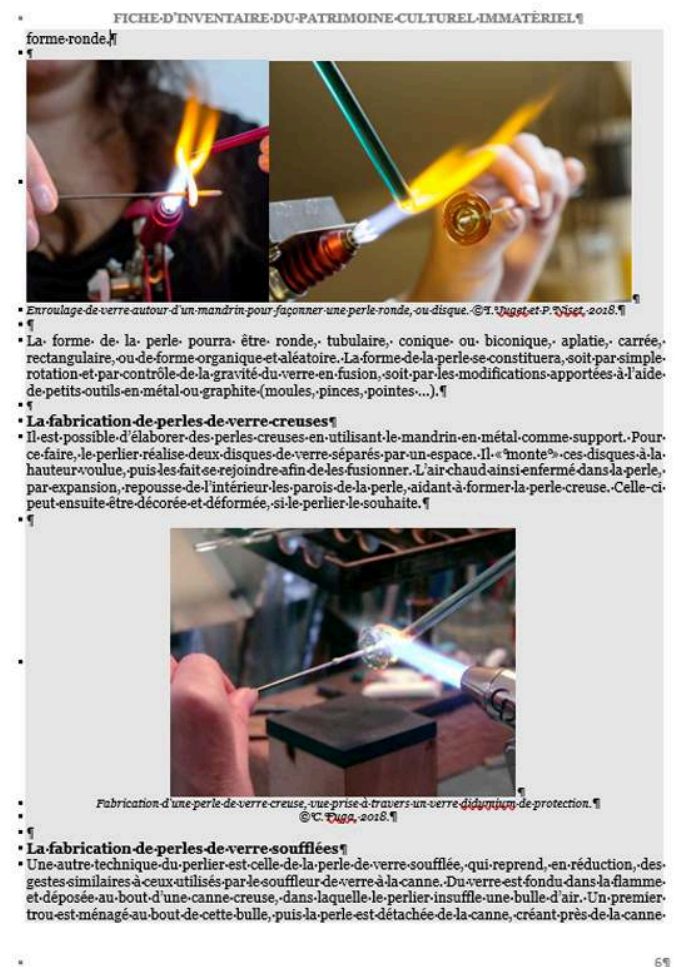

Technique de réalisation des perles de verre, description illustrée extraite de la fiche «L'artisanat de la perle de verre en France », Inventaire national du patrimoine culturel immatériel, Paris, ministère de la Culture, 2018, p. 6. 
Figure 7

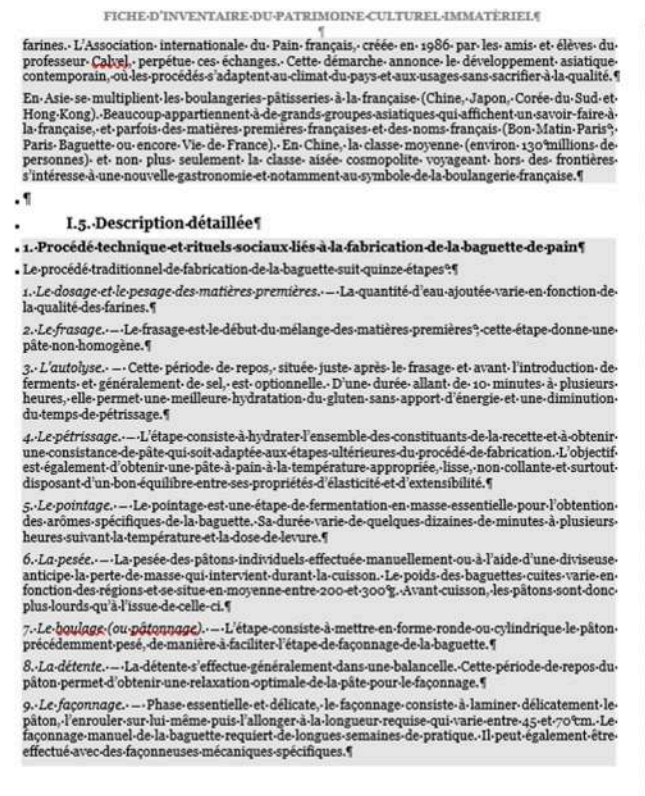



Technique de fabrication artisanale d'une baguette de pain, description illustrée extraite de la fiche "Les savoir-faire artisanaux et la culture de la baguette de pain », Inventaire national du patrimoine culturel immatériel, Paris, ministère de la Culture, 2018, p. 5-6.

12 L'évocation des outils, à l'appui des gestes techniques, fait l'objet soit d'énumérations, parfois longues, comme dans la fiche " Les savoir-faire du couvreur zingueur parisien " [fig. 8], par exemple, qui les répartit entre instruments de mesure, de traçage, de pliage, de découpe du zinc, de façonnage et de soudure; soit d'un recours à l'illustration photographique, parfois légendée avec précision [fig. 9]. 
Figure 8

\begin{abstract}
Sur l'un des côtés du plateau de la plieuse sont fixées les deux pinces à màchoires plates, qui
permettent de faire un ourlet sur un ou plusieurs côtés de la feuille de zinc. Ces deux pinces sont actionnees par deux leviers que le couvreur soulève dans un geste puissant et rapide. La première levée sert a faire une pliure à $90^{\circ}$; la seconde levée permet d'aplatir la pliure pour en faire un ourlet plat.

Sur le côte opposé du plateau de la plieuse est fixee la corniere en fer. Elle court sur toute la longueur du plateau. Elle permet de façonner la feuille de zinc a la batte de bois (pour réalisation d'une noue à l'intersection de deux versants, par exemple).
\end{abstract}

Les outils de decoupe du zinc

- La cisaille " pelican », appelée aussi « cisaille passe-feuille "

- La cisaille " bichantourneuse ";

- La tracette : cet outil en acier découpé est très pratique, car il permet de se dispenser de la règle, pour tracer des lignes distantes de quelques centimetres du bord d'une feuille de zinc. Chaque cran correspond à une mesure. Le cran place contre le bord de la feuille guide parallelement l outil, tandis que le bec trace sur le zinc la ligne qui va marquer le pli. Les à la batte en bois

- La griffe a zinc: pourne d une pointe a carbure de tungstène, elle permet de tracer un trait passer plusieurs fois la griffe sur le trait. A chaque passage, la griffe enlève un copeau de zinc. Pour couper la feuille, il suffit ensuite de la plier sur le bord de l'établi

- La scie à métaux.

Les outils de façonnage

- La batte en bois : elle sert à façonner le zinc. Le compagnon a place sa feuille de zinc sur l'établi, du côte de la corniere en fer qui presente une arete vive. Munı de sa batte, souven gainée de cuir, il frappe le rebord de la feuille sur toute sa longueur, afin de la rabattre à angle droit sur la comiere

- La pince à ourlet,

- La pince à border plate ou coudée.

Les outilis de soudure

- Le fer à souder : constitué d'une panne (tête) en cuivre rouge et alimenté par une bonbonne de gaz

- La lime pour nettoyer la panne du fer à souder:

- Un bloc de sel ammoniac pour étamer la panne du fer à souder en la frottant

- La targette : barrette de métal constituée de $1 / 3$ d'étain et de $2 / 3$ de plomb :

- Petite caisse en bois contenant le nécessaire à souder : bloc de sel d'ammoniac, peti récipient d'acide chlorhydrique, petit pinceau, éponge

Énumération des outils spécifiques pour le façonnage et la pose du zinc. Extrait de la fiche «Les savoir-faire du couvreur zingueur parisien », Inventaire national du patrimoine culturel immatériel, Paris, ministère de la Culture, 2017, p. 9-10.

Figure 9

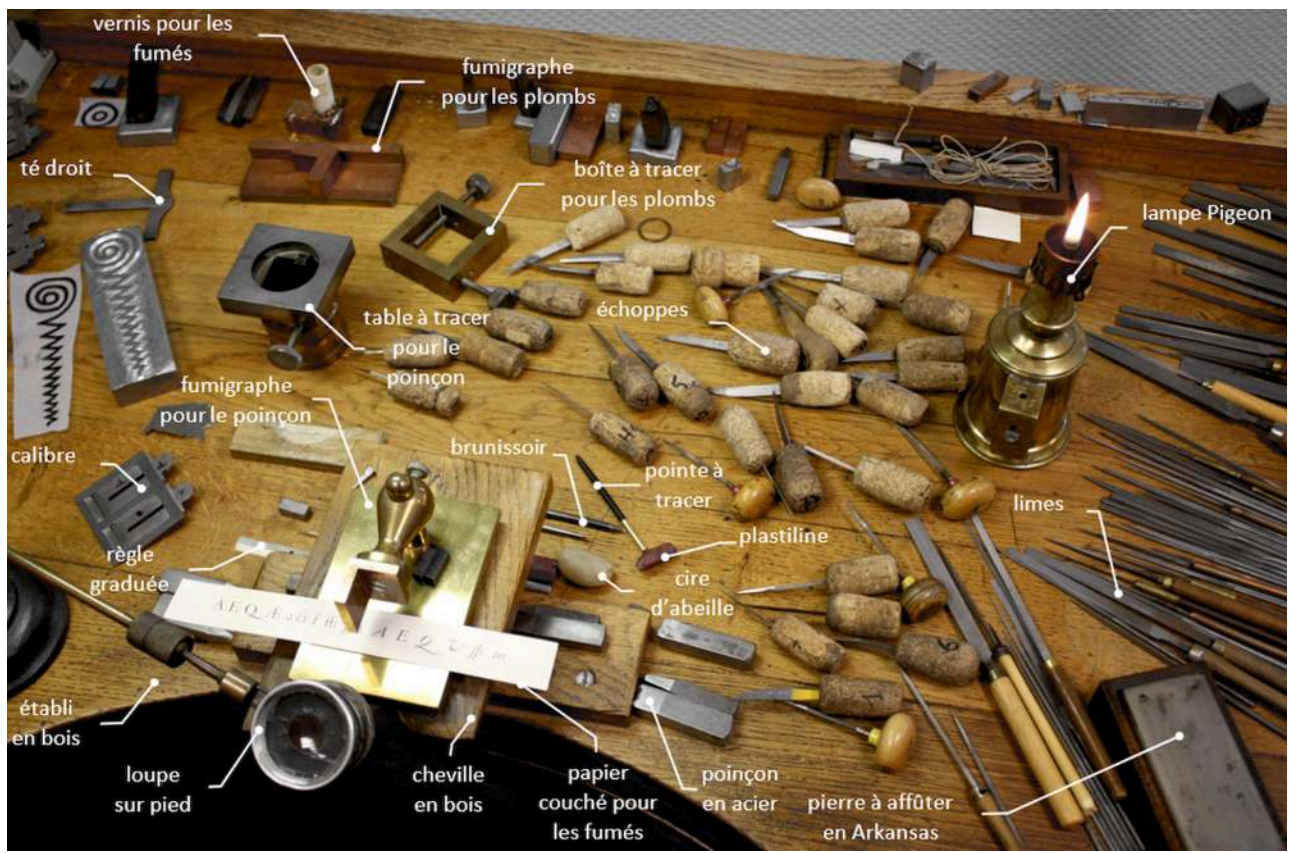

Outillage de la gravure de poinçons typographique. Vue extraite de la fiche « La gravure des poinçons typographiques », Inventaire national du patrimoine culturel immatériel, Paris, ministère de la Culture, 2018, p. 12

Photo : Nelly Gable / cliché : Imprimerie nationale. 
13 La fiche d'inventaire prévoit la citation de ressources à l'appui. La bibliographie est ici l'occasion de citer les manuels techniques et des revues professionnelles ${ }^{5}$. La filmographie renvoie vers des films documentaires, courts mais didactiques, en libre accès (sur les plateformes YouTube, Viméo, Dailymotion), qui seront, sur le site du ministère de la Culture, cités par un hyperlien en fin de fiche ( $c f$. annexe 1, partie IV.2), et, sur la plate-forme collaborative PCI Lab, signalés par une icône dès l'ouverture de la fiche invitant l'internaute à les consulter dès l'amont. La sitographie énumère souvent les sites internet des praticiens, des entreprises, des organisations professionnelles (associations, syndicats, groupements), des centres de formation, des musées des techniques, voire des fournisseurs spécialisés.

\section{Une écriture soumise à un champ de valeurs}

Imposant un procédé d'écriture très spécifique des savoir-faire techniques ou artisanaux, les champs descriptifs de la fiche-type d'inventaire des pratiques, transmise aux porteurs de projet, rendent compte, par leur teneur et leur organisation progressive, de ces quatre enjeux déjà cités, qui font de la description d'une technique, dans ses dimensions éthiques et opératives, un item d'un instrument patrimonial plus large : sélection de pratiques au regard de critères d'éligibilité, méthode d'inventaire de type anthropologique, association des praticiens dans la conduite de l'inventaire, part importante donnée à la mesure de la viabilité et de la transmission des pratiques.

En plus de la forme revêtue, celle d'un support de synthèse, de 20 à 25 pages, compréhensible par des publics diversifiés, un champ de valeurs est donc très nettement appliqué au fait technique, objet de "transferts culturels et conceptuels ${ }^{6}$ ». À cet égard, les dossiers de candidature, accompagnés de dix photographies maximum et de films de huit à dix minutes maximum chacun, proposés en ligne sur le site de l'Unesco, constituent, par leur teneur sur les pratiques immatérielles concernées, un mode d'écriture encore bien davantage encadré, notamment par les limitations imposées au nombre de mots. On consultera ainsi les dossiers des six pratiques culturelles reposant directement sur un savoir technique inscrites à ce jour sur la Liste représentative du PCI de l'humanité : «La tapisserie d'Aubusson », «La tradition du tracé dans la charpente française » [fig. 10], «Le savoir-faire de la dentelle au point d'Alençon » et «Le compagnonnage, réseau de transmission de savoirs et des identités par le métier ", "Les savoir-faire liés au parfum en pays de Grasse " et "L'art de la construction en pierre sèche $»^{7}$. 
Figure 10

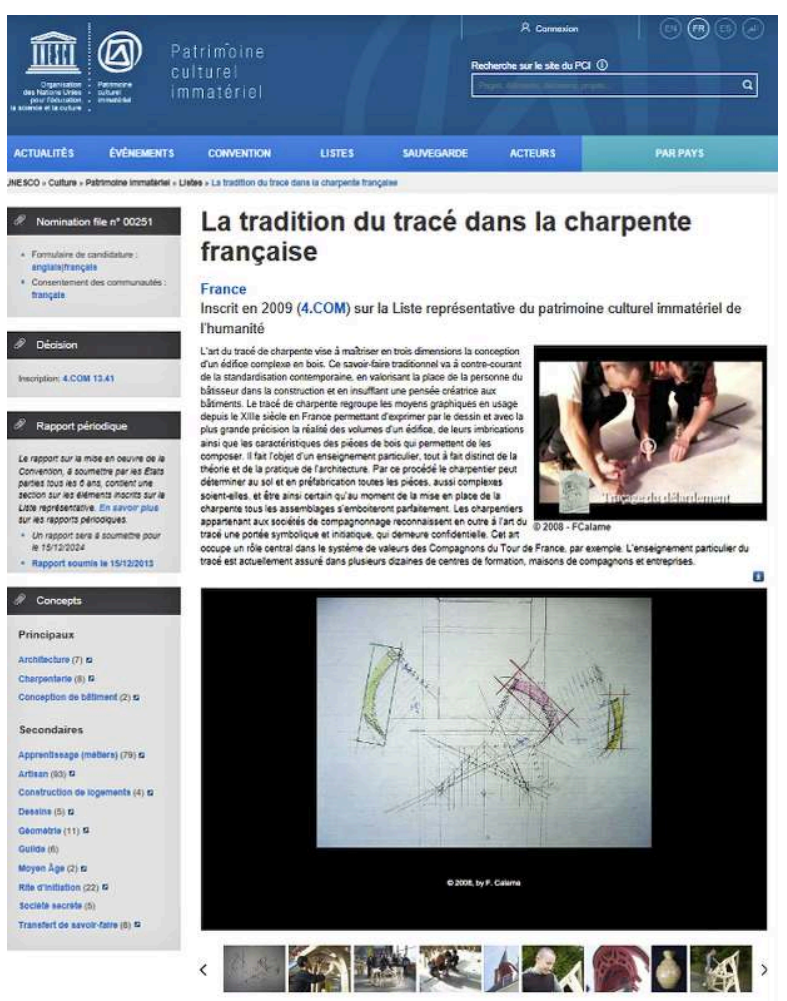

Capture d'écran de la page de l'élément « La tradition du tracé dans la charpente française » (2009) extraite du site internet de l'Unesco : accès au formulaire ICH-02, aux dix vues et au film associé (en ligne : https://ich.unesco.org/fr/RL/la-tradition-du-trace-dans-la-charpente-francaise-00251).

L'Inventaire national intègre la description synthétique de chaînes opératoires (la gravure de poinçons typographiques, la réalisation de toits en zinc à Paris, la fabrication de perles de verre...), mais conçoit chacune d'entre elle comme un système technique, en privilégiant l'approche par l'anthropologie des techniques, ce dont se ressentent beaucoup moins d'autres systèmes d'inventaire patrimoniaux. La méthode d'observation et d'analyse du PCI prend en compte autant les opérateurs que la conduite et le résultat du processus technique, en convoquant trois approches : 1/ la collecte et l'analyse des processus techniques (description, photographies, films); 2/ l'analyse des phénomènes sociaux et culturels (étude de la communauté associée, de ses pratiques sociales, avec une dimension plus anthropologique plus qu'historique, archivistique ou bibliographique); 3/la dialectique de leurs rapports mutuels, appliquée d'abord aux notions de transmission et de sauvegarde.

Dans certains cas, par ailleurs, la teneur même des informations ainsi exposées et structurées est influencée par la situation de concurrence professionnelle dans laquelle évoluent les détenteurs de traditions dans ce domaine spécifique (maitres d'art, artisans d'art, chefs de petites et moyennes entreprises...) : des tours de main, des gestes, des savoirs peuvent demeurer dans la sphère du secret ou du moins faire l'objet de descriptions rapides et finalement relativement superficielles, sans oublier les quelques demandes de retrait d'informations de fiches mises en ligne déjà intervenues depuis 2008.

18 La mise par écrit de ce fait technique résulte aussi de la prise de conscience d'une fragilité, quelle qu'en soit la cause. De fait, la nécessité de produire une telle fiche, 
surtout lorsque sa réalisation est proposée à la direction générale des Patrimoines de façon spontanée, et non dans le cadre de l'appel à projet annuel en recherche appliquée, répond le plus souvent à une situation de rupture (étiage démographique des pratiquants, crise économique de la structure, impacts d'évolutions sociétales plus amples...). La fiche réalisée rend donc compte, de façon sensible, d'une approche réflexive sur la viabilité des savoir-faire techniques décrits. Ainsi, l'adaptation de la technique à l'évolution des usages et des contextes est un aspect central, et conforme à la définition unesquienne :

Ce patrimoine culturel immatériel, transmis de génération en génération, est recréé en permanence par les communautés et les groupes, en fonction de leur milieu, de leur interaction avec la nature et de leur histoire, et leur procure un sentiment d'identité et de continuité, contribuant ainsi à promouvoir le respect de la diversité culturelle et la créativité humaine. (Convention 2003, art. 2.)

19 Comme les autres catégories, le patrimoine technique, fragile, est susceptible d'une adaptation en permanence pour éviter le risque de dégradation irréversible - une approche déterminée et déterminante que l'on a pu reprocher au Patrimoine culturel immatériel : "Réinventer le réel à travers la visualisation ou la transmission du passé d'entités censées être en voie de disparition ${ }^{8}$.» Dans cette mise en avant du caractère dynamique, créatif, adaptable de la pratique culturelle immatérielle, dans la capacité des communautés de trouver une réponse face aux aléas du monde environnant, résonne la définition toute spécifique du patrimoine qu'avait donnée Henry Ollagnon en son temps : «Un ensemble d'éléments matériels et immatériels qui concourent à sauvegarder l'autonomie et l'identité de leur titulaire et son adaptation face à un univers imprévisible ${ }^{9}$.»Et, dans cette même dimension de relation au passé, l'on pourrait appliquer parfaitement au processus de description du fait technique dans la perspective d'une inclusion à l'Inventaire national du patrimoine culturel immatériel les trois modalités déjà proposées pour qualifier le rapport du langage à la technique : une modalité prospective, une modalité descriptive et une modalité nostalgique ${ }^{10}$.

Dans la multitude de modes d'écriture que connaît le fait technique aujourd'hui, l'inventaire des pratiques culturelles immatérielles convoque très nettement l'anthropologie impliquée, appliquée ou collaborative, en analysant les techniques à travers leurs interactions sociales, culturelles et environnementales. En cela, il profite de l'ancrage ancien, dans la tradition anthropologique française, de la «technologie culturelle ", qui s'intéresse aux gestes techniques accompagnant la fabrication et la manipulation des objets ${ }^{11}$, avec la nécessité de considérer les objets matériels dans l'action, quand la tradition anglaise les a plutôt envisagés comme des spécimens de culture (material culture). Dans les fiches d'inventaire de pratiques techniques, les objets matériels, nécessairement très présents, sont les corrélats d'une action individuelle et collective, qui replacent dans un contexte particulier le geste qui anime l'objet et la chaîne opératoire qui lui donne son sens : qui sont ses producteurs? comment sont-ils organisés? quelle représentation se font-ils de leurs savoir-faire? quelle conscience ont-ils de sa fragilité ? quel sens donnent-ils à sa transmission ?, etc. Cette convocation des objets, même dans un instrument portant sur le patrimoine immatériel, a été relevée déjà dans son caractère inévitable :

L'antinomie fondatrice, revendiquée d'ailleurs comme un véritable outil heuristique, consiste, alors, dans l'exigence de raccorder cette logique symbolique à des indices concrets ou à des traces matérielles, qui puissent simultanément figer, conserver, rendre explicite et transmissible la fluidité «immatérielle» des biens identifiés comme patrimoine de l'humanité ${ }^{12}$. 
Mais en coexposant dans une même définition fondatrice «les pratiques, représentations, expressions, connaissances et savoir-faire", d'une part, et «les instruments, objets, artefacts et espaces culturels qui leur sont associés ", d'autre part, les concepteurs de la Convention de 2003 ont amené à réarticuler le matériel (bâti, outils, objets) et l'immatériel (gestes, rituel, discours, transmission), et pas seulement comme une nouvelle étape de la confrontation entre anthropologie des techniques et culture matérielle.

Loin de se cantonner à la seule technique, l'approche du fait technique par le PCI propose ainsi, dans une continuité des savoir-faire aux savoirs et aux représentations, une écriture intégrée de tous les faits, sociaux, techniques et culturels, à la manière dont Robert Cresswell ${ }^{13}$ déjà faisait des techniques des faits sociaux pleins et entiers, cherchant finalement d'abord à démontrer son caractère opératif [fig. 11 ; fig. 12].

Figure 11

\section{Formation technique Génie-végétal et Artisanat}

\section{Samedi 17 et Dimanche 18 - Février 2018 à la ferme Nissibart - 64130 BARCUS}

\footnotetext{
- Plessage de haies champêtres: comment créer des clôtures végétales vivantes et productives

- Vannerie sauvage : confectionner ses paniers pour la cueillette et le stockage à partir de végétaux ligneux champêtres
}

Deux journées de stage de pratiques complémentaires, dédiées à l'apprentissage de Savoir-faire répertoriés au patrimoine culturel immatériel de France

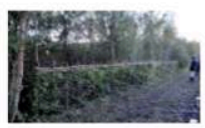

A destination

des professionnels de la nature (paysagistes, gestionnaires et exploitants d'espaces naturels) et des particuliers

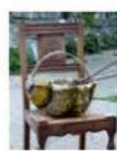

Renseignements et inscriptions (obligatoires) au 0547860008

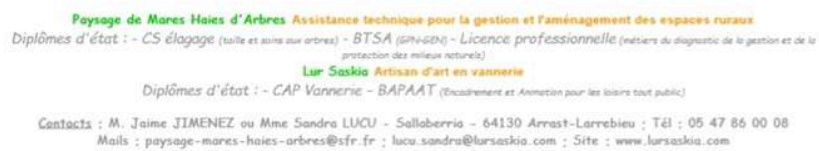

Un aboutissement du caractère opératif de l'Inventaire national : annonce d'un stage de formation en génie végétal et artisanat de l'association Paysage de Mares Haies d'arbres, conçu à partir de deux éléments techniques inscrits comme patrimoine culturel immatériel en France.

Cliché : Lur Saskia. 
Figure 12

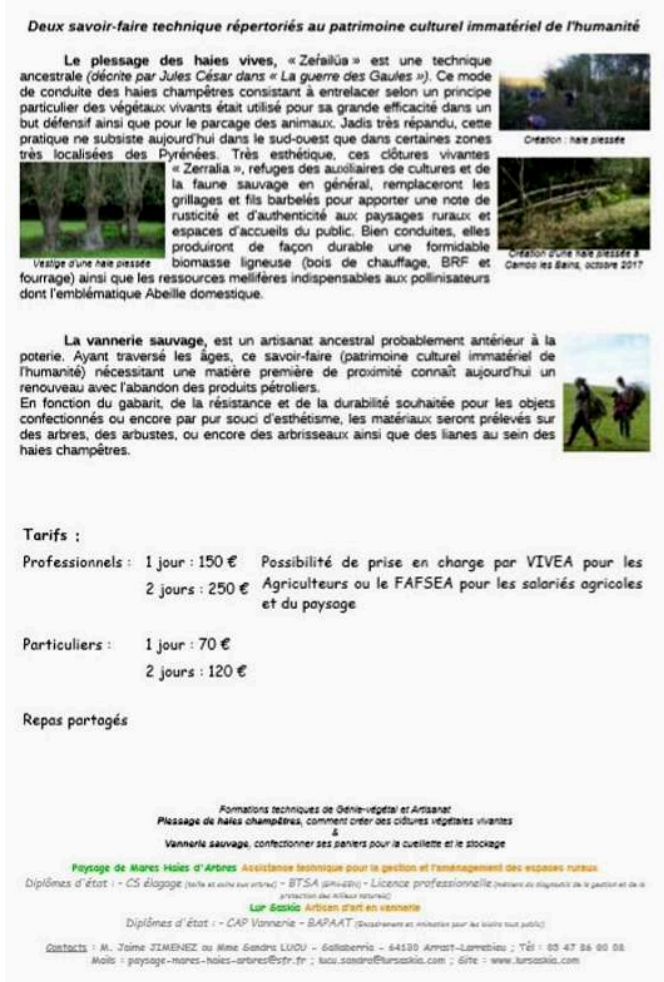

Détail de l'annonce du stage de formation en génie végétal et artisanat de l'association Paysage de Mares Haies d'arbres, conçu à partir des éléments techniques inscrits comme patrimoine culturel immatériel en France que sont le Plessage des haies vives et la Vannerie sauvage.

Cliché : Lur Saskia.

\section{ANNEXES}

\section{ANNEXE 1}

Champs de la fiche-type pour l'inventaire du patrimoine culturel immatériel (2019)

NOM DE L'ÉLÉMENT

[3 vues d'illustration, légendées et créditées]

Description sommaire [1500 caractères (espaces compris) maximum]

I. IDENTIFICATION DE L'ÉLÉMENT

I.1. Nom [150 caractères (espaces compris) maximum]

En français

En langue vernaculaire 
I.2. Domaine(s) de classification, selon l'Unesco

I.3. Communauté(s) et groupe(s) associé(s) à la pratique

I.4. Localisation physique

Lieu(x) de la pratique en France

Pratique similaire en France et/ou à l'étranger

I.5. Description détaillée [25 à 30000 caractères (espaces compris) maximum, texte illustré de photographies si nécessaire]

I.6. Langue(s) utilisée(s) dans la pratique

I.7. Éléments matériels liés à la pratique

Patrimoine bâti

Objets, outils, matériaux supports

II. APPRENTISSAGE ET TRANSMISSION DE L'ÉLÉMENT

II.1. Modes d'apprentissage et de transmission

II.2. Personnes/organisations impliquées

III. HISTORIQUE

III.1. Repères historiques [10 000 caractères (espaces compris) maximum]

III.2. Évolution/adaptation/emprunts de la pratique

IV. VIABILITÉ DE L'ÉLÉMENT ET MESURES DE SAUVEGARDE

IV.1. Viabilité de la pratique

IV.2. Mise en valeur et mesures de sauvegarde existantes

Modes de sauvegarde et de valorisation

Actions de valorisation à signaler

Modes de reconnaissance publique

IV.3. Mesures de sauvegarde envisagées à l'avenir

IV.4. Documentation à l'appui

Récits liés à la pratique et à la tradition

Inventaires réalisés liés à la pratique

Bibliographie sommaire

Filmographie sommaire

Sitographie sommaire

V. PARTICIPATION DES COMMUNAUTÉS, GROUPES ET INDIVIDUS

V.1. Praticien(s) rencontré(s) et contributeur(s) de la fiche

Nom(s) / Fonction(s) / Coordonnées

V.2. Soutiens et consentements reçus 


\section{MÉTADONNÉES DE GESTION}

VI.1. Rédacteurs(s) de la fiche

$\operatorname{Nom}(s)$ / Fonction(s) / Coordonnées

VI.2. Enquêteur(s), chercheur(s) ou membre(s) du comité scientifique associé

$\operatorname{Nom}(s) /$ Fonctions

Lieux $(x)$ et date/période de l'enquête

VI.3. Données d'enregistrement

Date de remise de la fiche

Année d'inclusion à l'inventaire

$N^{\circ}$ de la fiche

Identifiant ARKH

Autres livrables attendus :

- lettres de consentement des membres de la communauté,

- lettres de soutien,

- au moins 3 vues d'illustration,

un court film documentaire (facultatif).

\section{NOTES}

1. http://www.culture.gouv.fr/Thematiques/Patrimoine-culturel-immateriel/L-inventairenational/Inventaire; l'Inventaire national est complété par des inventaires thématiques ou géographiques tenus par des structures de statuts divers (collectivités territoriales, associations, ethnopôles labellisés du ministère...) à l'échelle du territoire, énumérés dans le « Répertoire des inventaires du patrimoine culturel immatériel »: http://www.culture.gouv.fr/Thematiques/ Patrimoine-culturel-immateriel/L-inventaire-national/Le-repertoire-des-inventaires

2. http://pcilab-new.huma-num.fr/ ; la plateforme PCI Lab fait l'objet du projet Wikipatrimoine de recherche en sciences de l'information et de la communication, financé en 2017-2018 par le LabEx Les Passés dans le présent, la Comue Université Paris-Lumière et la direction générale des Patrimoines (DPRPS).

3. CHAVE Isabelle, «Les savoir-faire du bâti traditionnel : un champ pour le patrimoine culturel immatériel », Maisons paysannes de France, $\mathrm{n}^{\circ}$ 207, 2018.

4. MERMET Gilles, «Les savoir-faire du couvreur zingueur parisien », Paris, ministère de la Culture (Inventaire national du Patrimoine culturel immatériel), 2017, p. 3-9.

5. Par exemple, pour la fiche "L'art de la construction en pierre sèche ", incluse en 2010 à l'Inventaire national du PCI : CAPEB, ABPS, MURAILLERS DE PROVENCE et al., Guide de bonnes pratiques de construction de murs de soutènement en pierre sèche, Lyon, École nationale des travaux publics de l'État, 2008 ; COSTE Pierre (dir.), Pierre sèche, Manosque, Le Bec en l'air, 2008 ; CETTOUR Denis, «Les restanques: une technique bien rodée", Pierre actual, n 857, 2008, p. 59-60. Par ailleurs, pour un point complet sur ce sujet, voir : CHAVE Isabelle \& CORNU Claire, « La pierre sèche : de la recherche appliquée à la reconnaissance internationale », Monumental, 2009-1, «La pierre dans l'architecture : conservation, restauration, création », 2019, p. 24-25.

6. BONDAZ Julien, «Entrer en collection. Pour une ethnographie des gestes et des techniques de collecte », Les Cahiers de l'École du Louvre, $\mathrm{n}^{\circ}$ 4, «Les modalités de la collecte : rapt, troc, marché, 
fouilles, don... Et leur impact sur l'objet », 2014 [en ligne], https://journals.openedition.org/cel/ 472 [lien valide en août 2018].

7. «La tapisserie d'Aubusson» (2009): https://ich.unesco.org/fr/RL/la-tapisseriedaubusson-00250. «La tradition du tracé dans la charpente française» (2009): https:// ich.unesco.org/fr/RL/la-tradition-du-trace-dans-la-charpente-francaise-00251. " Le savoir-faire de la dentelle au point d'Alençon » (2010) : https://ich.unesco.org/fr/RL/le-savoir-faire-de-ladentelle-au-point-dalencon-00438. « Le compagnonnage » (2010) : https://ich.unesco.org/fr/RL/ le-compagnonnage-reseau-de-transmission-des-savoirs-et-des-identites-par-le-metier-00441.

«Les savoir-faire liés au parfum en pays de Grasse »: https://ich.unesco.org/fr/RL/les-savoirfaire-lies-au-parfum-en-pays-de-grasse-la-culture-de-la-plante-a-parfum-la-connaissance-desmatieres-premieres-naturelles-et-leur-transformation-l-art-de-composer-le-parfum-01207 (2018). « L'art de la construction en pierre sèche » (2018) : https://ich.unesco.org/fr/RL/l-art-dela-construction-en-pierre-seche-savoir-faire-et-techniques-01393. Les éléments français ainsi reconnus sont au nombre de 18 au $1^{\mathrm{er}}$ janvier 2020.

8. CIARCIA Gaetano, "La perte durable. Étude sur la notion de "patrimoine immatériel" ", Les Carnets $d u$ Lahic, $\mathrm{n}^{\circ} 1,2006$, p. 6 [en ligne], https://www.iiac.cnrs.fr/IMG/pdf/ Ciarcia_perte_durable-2.pdf [lien valide en juin 2020].

9. OLLAGNON Henri, «Acteurs et patrimoine dans la gestion de la qualité des milieux naturels ", Aménagement et nature, $\mathrm{n}^{\circ} 74,1984$, p. 118-127 ; repris par MONTGOLFIER Jean de, «La gestion patrimoniale des ressources naturelles ", in JEUDY Henri-Pierre (dir.), Patrimoines en folie, textes issus du séminaire organisé au Collège international de philosophie (Paris, 1987-1988), Paris, Éditions de la Maison des sciences de l'homme, coll. «Ethnologie de la France », 1990, p. 21-27.

10. DUARTE Rodrigues A., « Mémoire et technique », in JEUDY Henri-Pierre (dir.), Patrimoines en folie, textes issus du séminaire organisé au Collège international de philosophie (Paris, 1987-1988), Paris, Éditions de la Maison des sciences de l'homme, coll. « Ethnologie de la France », 1990, p. 251-258.

11. DIGARD Jean-Pierre, «La technologie en anthropologie: fin de parcours ou nouveau souffle?", L'Homme, n 19, vol.1, p.73-104, 1979. Disponible en ligne, persee.fr/doc/ hom_0439-4216_1979_num_19_1_367929 [lien valide en août 2018]; DIGARD Jean-Pierre, «Anthropologie des techniques et anthropologie cognitive», Études rurales, n 169-170, «Transmissions », 2004. Disponible en ligne, journals.openedition.org/etudesrurales/3007 [lien valide en août 2018].

12. CIARCIA Gaetano, "La perte durable. Étude sur la notion de "patrimoine immatériel" ", Op. cit., p. 5.

13. CRESSWELL Robert, Prométhée ou Pandore? Propos de technologie culturelle, Paris, Kimé, coll. «Anthropologies », 1996.

\section{RÉSUMÉS}

Créé en 2008 à la suite de l'approbation par la France de la Convention Unesco pour la sauvegarde du patrimoine culturel immatériel (PCI) (2003), l'Inventaire national du patrimoine culturel immatériel est tenu par le ministère de la Culture (direction générale des Patrimoines). Avec près de $25 \%$ de fiches relatives aux savoir-faire de l'artisanat traditionnel, il constitue une nouvelle approche patrimoniale du fait technique : mise en œuvre des matériaux, techniques agricoles, culinaires, maritimes ou halieutiques, techniques artistiques, techniques du bâti traditionnel ou 
$\mathrm{du}$ textile, facture instrumentale. Aux côtés d'autres outils documentaires du patrimoine culturel, l'inventaire du PCI donne une orientation spécifique: une sélection de pratiques techniques qui doivent répondre aux dispositions et aux critères de la Convention; une démarche d'inventaire de nature anthropologique, décrivant savoirs, techniques et savoir-faire par le prisme des communautés impliquées (professionnels, entreprises, organismes spécialisés...) ; autant d'importance donnée à la description des processus qu'à la viabilité des pratiques, aux conditions de transmission, à l'éducation formelle et non formelle, aux parcours de formation et aux éventuels systèmes de certification professionnelle. Du fait de l'analyse des techniques à travers leurs interactions sociales, culturelles et environnementales et du recours aux dimensions éthiques et opératives, l'écriture du fait technique se trouve ici très nettement soumise à un champ de valeurs.

Created in 2008 after the French approval of the UNESCO Convention for the Safeguarding of the Intangible Cultural Heritage (2003), the ICH National Inventory is kept by the Ministry of Culture (Heritage Directorate). With almost $25 \%$ inventory files regarding traditional craftsmanship, it provides a new heritage approach of techniques: processing of materials, agricultural, culinary, maritime, fishery techniques, artistic techniques, traditional building techniques, textile techniques, musical instrument making. Together with other documentary tools for cultural heritage, the ICH inventory gives a specific orientation: a selection of technical practices which must meet the provisions and criteria of the Convention, an inventorying process of an anthropological nature, which describes knowledge, techniques and know-how through the lens of the communities involved (practitioners, companies, specialized organisations...) and the same importance given to the process mapping as to the viability of practices, transmission conditions, formal and non-formal education, training pathways and any professional certification system. By analysing techniques through their social, cultural and environmental interactions and by using ethical and operative dimensions, the writing of the technical fact is here very clearly subjected to a value field.

\section{INDEX}

Mots-clés : anthropologie des techniques, chaîne opératoire, patrimoine culturel immatériel, processus technique, savoir-faire artisanaux, transmission des savoirs

Keywords : anthropology of techniques, operating chain, intangible cultural heritage, technical process, craftsmanship, knowledge transmission

\section{AUTEUR}

\section{ISABELLE CHAVE}

Conservateur en chef du patrimoine, adjointe au chef du département du Pilotage de la recherche et de la Politique scientifique, direction générale des Patrimoines, ministère de la Culture.

isabelle.chave@culture.gouv.fr 